\title{
The Saturation Throughput Region of $p$-Persistent CSMA
}

\author{
Yi Gai, Shankar Ganesan, and Bhaskar Krishnamachari \\ Ming Hsieh Department of Electrical Engineering \\ Viterbi School of Engineering, University of Southern California \\ Los Angeles, CA 90089 \\ \{ygai, shankarg, bkrishna@usc.edu\}
}

\begin{abstract}
Many modern wireless data networks employ Carrier Sense Multiple Access (CSMA) for efficient medium access. The $p$-persistent CSMA protocol is an analytically tractable version of CSMA that has been used successfully to model practical medium access protocols such as the IEEE 802.11 Distributed Coordination Function (DCF). We present a closedform expression to characterize the access probabilities at the boundary of the saturation throughput region of $p$-persistent CSMA. This expression is a non-trivial generalization of the elegant result, obtained by J. Massey and P. Mathys in 1985, that the boundary of the saturation throughput region for slotted Aloha corresponds to the users adopting independent access probabilities that sum up to 1 . We also present a closed form expression for the throughput values obtained at the boundary of the saturation throughput region of $p$-persistent CSMA for the case of 2 users.
\end{abstract}

\section{INTRODUCTION}

Carrier Sense Multiple Access (CSMA) is a basic access mechanism used for efficient medium access in wireless networks. It forms the basis of the medium access control (MAC) protocols for many wireless network standards, such as IEEE 802.11 [1] and IEEE 802.15.4 [2].

The $p$-persistent CSMA algorithm is a simple version of CSMA, first proposed in [3]. In homogeneous $p$-persistent CSMA, users synchronously contend for access to the medium with a common access probability $p$, whenever it is free. The memoryless nature of this access model makes it the most analytically tractable version of CSMA, and it has therefore been widely studied, e.g., in [4], [5], [6], [7], [8], [9], [10], [11]. It has been shown in [5], [6] that a $p$-persistent CSMA protocol closely approximates the commonly-implemented version of the CSMA protocol used in IEEE 802.11 (which employs uniform backoff counters and binary exponential backoff) if the value of $p$ is chosen such that the average backoff intervals of the two protocols are the same. For this reason, key results on analyzing the saturation throughput of IEEE 802.11 and enhancing its performance [5], [6], [10], have used this $p$ persistent CSMA model as their basis.

Bianchi [5] proposed to analyze the backoff process of IEEE 802.11 with a discrete-time Markov chain model, and provided an analytical model to compute the saturation throughput, using the saturation throughput performance of $p$-persistent

This research was sponsored in part by the U.S. National Science Foundation under Award CNS-1049541.
CSMA as an approximation. Calì et al. [6] derived the optimal value of $p$ for maximizing the throughput of p-persistent CSMA, and used it to develop a distributed algorithm running at each user to tune its backoff. Bruno et al. [10] similarly analyze the optimal value of $p$ for maximizing the throughput and minimizing the energy. All of these prior works use the homogeneous p-persistent CSMA model, which assumes that all users employ the same $p$, in which case all users have the same expected throughput.

A natural generalization of the standard homogeneous $p$ persistent CSMA algorithm is the heterogeneous p-persistent CSMA, in which different users are allowed to pick different values $p_{i}$. In this case, because users will get different channel access opportunities, the performance is characterized by a throughput region, which describes the set of all feasible throughput vectors which can be attained by different values of $p_{i}$. Such a model has been considered in a few recent works [12], [13], [14].

The focus of this paper is on the following fundamental question: what is the saturation throughput region for $p$ persistent CSMA? We answer this question by providing a closed-form expression that characterizes the vector of access probabilities at the Pareto boundary of the throughput region. We also provide an explicit characterization of the boundary of the throughput region in terms of rates for the special case of $n=2$ users.

Consider $n$ backlogged users contending for the medium. Let $T$ be the expected duration of packet transmission, and $\sigma$ be the length of the contention period. We prove that the boundary of the saturation throughput region for $p$-persistent CSMA corresponds exactly to the access probabilities $p_{i}$ satisfying the following relationship:

$$
1-\prod_{i}\left(1-p_{i}\right)+\frac{T}{\sigma}\left(\sum_{i} p_{i}+\prod_{i}\left(1-p_{i}\right)-1\right)=1 .
$$

Note that this is a necessary and sufficient condition for the boundary of the throughput region.

Interestingly, the well known slotted Aloha protocol [15] can be viewed as a special case of $p$-persistent CSMA, when $T=\sigma$. It was shown by Massey and Mathys in 1985 [16] that the boundary of the saturation throughput region for slotted Aloha corresponds exactly to the access probabilities summing to 1 , i.e. $\sum p_{i}=1$. Notice that our main result in equation (1) includes their finding as a special case. 
Massey and Mathys also showed that the boundary of the saturation throughput region for slotted Aloha for two users is characterized by the equation $\sqrt{S_{1}}+\sqrt{S_{2}}=1$ where $S_{1}$ and $S_{2}$ are the throughput values respectively for users 1 and 2. We also show in this paper that the corresponding generalization for $p$-persistent CSMA is that the throughput values at the boundary of the two-user throughput region satisfy the following condition:

$$
\sqrt{\frac{T}{\sigma}} \cdot\left(1-S_{1}-S_{2}\right)=2 \sqrt{S_{1} S_{2}} .
$$

\section{Problem Formulation}

We assume there are $n$ backlogged users in a heterogeneous $p$-persistent CSMA system. Time is slotted, and packets are only allowed to be transmitted as the beginning of each time slot. Users do not attempt to transmit when the channel is busy, but when it is idle, user $i$ transmits with access probability $p_{i}$. A transmission is successful when only one user transmits, else there is a collision. We assume that the average transmission duration is $T$, regardless of whether it is successful or a collision. Denote $S_{i}$ as the normalized saturation throughput for user $i$, defined as the asymptotic time-averaged proportion of the time that the channel is used for successful packet transmissions by user $i$.

The time between the end of one transmission and the start of another can be treated as a renewal epoch, and using the elementary renewal theorem [17], it can be shown that

$$
\begin{aligned}
S_{i} & =\frac{\mathbb{E}[\text { successful transmission time for } i \text { per epoch }]}{\mathbb{E}[\text { epoch duration }]} \\
& =\frac{p_{i} \prod_{j \neq i}\left(1-p_{j}\right) T}{\sigma \prod_{i}\left(1-p_{i}\right)+\left(1-\prod_{i}\left(1-p_{i}\right)\right) T}
\end{aligned}
$$

Denote the system saturation throughput vector as $\mathbf{S}=$ $\left(S_{1}, S_{2}, \ldots, S_{n}\right)$. Then the saturation throughput region $\mathcal{R}$ is defined as the set of all feasible $\mathbf{S}$.

The boundary of the throughput region $\mathcal{R}$ consists of the set of all Pareto efficient system throughput vectors. A throughput vector $S^{*}$ is said to be Pareto efficient if there exists no other throughput vector $S^{\prime} \in \mathcal{R}$ that offers a throughput improvement to at least one user without hurting any other users. For a throughput vector $\mathbf{S}^{*}$ to be Pareto efficient, for all $i, S_{i}^{*}$ must be the maximized value of the following optimization problem:

$$
\begin{array}{cl}
\max & S_{i} \\
\text { s.t. } & S_{j}=S_{j}^{*}, \forall j \neq i \\
& 0 \leq p_{i} \leq 1, \forall 1 \leq i \leq n
\end{array}
$$

Thus every point on the boundary of the throughput region, $\mathbf{S}^{*}$, represents the simultaneous solution of $n$ interdependent optimization problems.

\section{AnAlysis of SATURATion Throughput Region}

Our key contribution is providing an simple and elegant expression for the boundary of the saturation throughput region (the Pareto frontier), as indicated in Theorem 1 below. The key mathematical challenge we encounter and overcome is solving the $n$ simultaneous optimization problems in (4), which each require the constrained maximization of implicit functions.

Theorem 1: At, and only at, all points on the boundary of the saturation throughput region for $p$-persistent CSMA, the access probabilities satisfy the condition in equation (1).

Proof: Note that if there exists any user with a access probability 1 , this will force the throughput of all the other stations to be 0 . This case, which corresponds to the corners of the boundary of the throughput region, trivially satisfies the given condition. So in the following, we focus on the case when $p_{i}<1$ for all users. Note also that if there exist any users with $p_{i}=0$, then they are effectively absent from the system and neither receive any throughput nor have any effect on the condition in equation (1); for simplicity, we therefore restrict our focus to users with non-zero access probability in the following, so that $n$ refers to the total number of users with non-zero access probability.

First, we prove that if the throughput vector $\mathbf{S}$ is at the Pareto boundary, then the condition in equation (1) holds.

From equation (3), we have

$$
\frac{S_{i}}{S_{j}}=\frac{p_{i}\left(1-p_{j}\right)}{p_{j}\left(1-p_{i}\right)}, \quad \forall i, j .
$$

Equation (5) implies

$$
p_{j}=\frac{S_{j} p_{i}}{S_{i}\left(1-p_{i}\right)+S_{j} p_{i}}, \quad \forall i .
$$

Substituting equation (6) into equation (3), the throughput of user $i$ can be written as

$$
\begin{aligned}
S_{i}= & \left(p_{i} \prod_{j \neq i} \frac{S_{i}\left(1-p_{i}\right)}{S_{i}\left(1-p_{i}\right)+S_{j} p_{i}} T\right) \\
& \cdot\left[\sigma\left(1-p_{i}\right) \prod_{j \neq i} \frac{S_{i}\left(1-p_{i}\right)}{S_{i}\left(1-p_{i}\right)+S_{j} p_{i}}\right. \\
& \left.+\left(1-\left(1-p_{i}\right) \prod_{j \neq i} \frac{S_{i}\left(1-p_{i}\right)}{S_{i}\left(1-p_{i}\right)+S_{j} p_{i}}\right) T\right]^{-1} .
\end{aligned}
$$

Further simplification of equation (7) yields

$$
\begin{aligned}
& \sigma\left(1-p_{i}\right) S_{i}^{n-1}\left(1-p_{i}\right)^{n-1}-p_{i} S_{i}^{n-2}\left(1-p_{i}\right)^{n-1} T \\
+ & {\left[\prod_{j \neq i}\left(S_{i}\left(1-p_{i}\right)+S_{j} p_{i}\right)-\left(1-p_{i}\right) S_{i}^{n-1}\left(1-p_{i}\right)^{n-1}\right] T } \\
\quad & =0
\end{aligned}
$$

Equation (8) defines an implicit function $\mathcal{F}\left(S_{i}, p_{i}\right)=0$ when given all $S_{j}, j \neq i$. Then the maximization problem is, fixing all $S_{j}, j \neq i$,

$$
\begin{array}{cl}
\max & S_{i} \\
\text { s.t. } & 0 \leq p_{i} \leq 1, \forall 1 \leq i \leq n
\end{array}
$$


Using the method of Lagrange Multipliers, we define

$$
\mathcal{L}=-S_{i}+\sum_{i} \lambda_{i}\left(-p_{i}\right)+\sum_{i} \lambda_{i}^{\prime}\left(1-p_{i}\right) .
$$

Applying KKT conditions, note that $p_{i} \neq 0, \forall i$, so $\lambda_{i}=0, \forall i$. Also note that $p_{i} \neq 1, \forall i$, so $\lambda_{i}^{\prime}=0, \forall i$.

Hence

$$
\frac{d \mathcal{L}}{d p_{i}}=\left.0 \Longleftrightarrow \frac{d S_{i}}{d p_{i}}\right|_{S_{j}, j \neq i}=0 .
$$

Note that,

$$
\left.\frac{d S_{i}}{d p_{i}}\right|_{S_{j}, j \neq i}=0 \Longleftrightarrow-\frac{\frac{\partial \mathcal{F}}{\partial p_{i}}}{\frac{\partial \mathcal{F}}{\partial S_{i}}}=0 \Longrightarrow \frac{\partial \mathcal{F}}{\partial p_{i}}=0
$$

Solving this implicit differentiation yields

$\mathcal{G}_{1}\left(S_{i}, p_{i}\right)-\mathcal{G}_{2}\left(S_{i}, p_{i}\right)-\mathcal{G}_{3}\left(S_{i}, p_{i}\right)+\mathcal{G}_{4}\left(S_{i}, p_{i}\right)=0$,

where,

$$
\begin{aligned}
& \mathcal{G}_{1}\left(S_{i}, p_{i}\right)=\sigma n\left(1-p_{i}\right)^{n-1} S_{i}^{n-1}, \\
& \mathcal{G}_{2}\left(S_{i}, p_{i}\right)=T\left[\sum_{j \neq i}\left(S_{j}-S_{i}\right) \prod_{k \neq j}\left(S_{i}\left(1-p_{i}\right)+S_{k} p_{i}\right)\right], \\
& \mathcal{G}_{3}\left(S_{i}, p_{i}\right)=T\left[n\left(1-p_{i}\right)^{n-1} S_{i}^{n-1}\right], \\
& \mathcal{G}_{4}\left(S_{i}, p_{i}\right)=T S_{i}^{n-2}\left(1-p_{i}\right)^{n-2}\left(1-n p_{i}\right) .
\end{aligned}
$$

Let

$$
\mathcal{H}\left(p_{i}\right)=\frac{\left(1-p_{i}\right) \prod_{j \neq i} p_{j}}{n p_{i}^{n-1} \prod_{j \neq i} S_{j}} .
$$

Then,

$\left(\mathcal{G}_{1}\left(S_{i}, p_{i}\right)-\mathcal{G}_{2}\left(S_{i}, p_{i}\right)-\mathcal{G}_{3}\left(S_{i}, p_{i}\right)+\mathcal{G}_{4}\left(S_{i}, p_{i}\right)\right) \mathcal{H}\left(p_{i}\right)=0$.

Now we calculate the expression of the four parts on the left hand side one by one. Using the definitions in (14)-(17) and (18), and equations (3) and (5), the following expressions can be derived:

$$
\begin{aligned}
\mathcal{G}_{1}\left(S_{i}, p_{i}\right) \mathcal{H}\left(p_{i}\right) & =\sigma \prod_{i}\left(1-p_{i}\right), \\
\mathcal{G}_{2}\left(S_{i}, p_{i}\right) \mathcal{H}\left(p_{i}\right) & =T\left(\frac{\sum_{i} p_{i}}{n p_{i}}-1\right), \\
\mathcal{G}_{3}\left(S_{i}, p_{i}\right) \mathcal{H}\left(p_{i}\right) & =T \prod_{i}\left(1-p_{i}\right), \\
\mathcal{G}_{4}\left(S_{i}, p_{i}\right) \mathcal{H}\left(p_{i}\right) & =-T \cdot \frac{\left(n p_{i}-1\right) \mathcal{A}\left(p_{i}\right)}{n p_{i}},
\end{aligned}
$$

where we define

$$
\mathcal{A}\left(p_{i}\right)=\frac{1}{T}\left[\sigma \prod_{i}\left(1-p_{i}\right)+\left[1-\prod_{i}\left(1-p_{i}\right) T\right]\right] .
$$

Now applying the result of equations (20)-(23) to equation (19), we get:

$$
\begin{gathered}
\sigma \prod_{i}\left(1-p_{i}\right)+\left(1-\prod_{i}\left(1-p_{i}\right)\right) T \\
=\frac{\sum_{i} p_{i}+\left(n p_{i}-1\right) \mathcal{A}\left(p_{i}\right)}{n p_{i}} T
\end{gathered}
$$

Note that equation (24) implies

$$
\sigma \prod_{i}\left(1-p_{i}\right)+\left(1-\prod_{i}\left(1-p_{i}\right)\right) T=\mathcal{A}\left(p_{i}\right) T .
$$

The left-hand sides of equations (25) and (26) are the same. So we have

$$
\begin{aligned}
& \frac{\sum p_{+}\left(n p_{i}-1\right) \mathcal{A}\left(p_{i}\right)}{n p_{i}} T=\mathcal{A}\left(p_{i}\right) T \\
& \Longrightarrow \mathcal{A}\left(p_{i}\right)-\frac{\sum_{i} p_{i}+\left(n p_{i}-1\right) \mathcal{A}\left(p_{i}\right)}{n p_{i}}=0 \\
& \Longrightarrow \frac{\mathcal{A}\left(p_{i}\right)-\sum_{i} p_{i}}{n p_{i}}=0 \\
& \Longrightarrow \mathcal{A}\left(p_{i}\right)=\sum_{i} p_{i} .
\end{aligned}
$$

Finally, we substitute equation (27) into equation (25), and we get

$$
\begin{aligned}
& \sigma \prod_{i}\left(1-p_{i}\right)+\left(1-\prod_{i}\left(1-p_{i}\right)\right) T=\frac{\sum_{i} p_{i}+\left(n p_{i}-1\right) \sum_{i} p_{i}}{n p_{i}} T \\
& \Longrightarrow \frac{T}{\sigma}\left[\sum_{i} p_{i}+\prod_{i}\left(1-p_{i}\right)-1\right]=\prod_{i}\left(1-p_{i}\right) \\
& \Longrightarrow 1-\prod_{i}\left(1-p_{i}\right)+\frac{T}{\sigma}\left(\sum_{i} p_{i}+\prod_{i}\left(1-p_{i}\right)-1\right)=1
\end{aligned}
$$

We have thus proved one direction, i.e., that if the throughput vector is at the Pareto boundary, it satisfies the condition in equation (1). We now consider the other direction, i.e., if the condition in equation (1) does hold, we prove that the corresponding throughput vector must be on the Pareto boundary.

This can be proved by contradiction. Assume there is an access probability vector $\mathbf{p}=\left(p_{1}, p_{2}, \ldots, p_{n}\right)$ that satisfies the condition in equation (1) that is not on the Pareto boundary. Then there must be at least one $S_{i}$ that can be increased while keeping the other $S_{j}, \forall j \neq i$ fixed, to get to a point on the Pareto boundary. However, given the form of the throughput functions $S_{i}$ as given in equation (3), it can be shown that this requires a strict increase in $p_{i}, \forall i$. However, it can also be shown that the left hand side of equation (1) is monotonic in all $p_{i}$. Therefore, such an increase will cause that left hand side to exceed 1, resulting in a point on the Pareto boundary that does not satisfy the condition in equation (1). This contradicts what we have already shown.

Given the above analysis of the conditions on the access probabilities, it is also possible to characterize the boundary of the throughput region in terms of the Pareto optimal throughput vectors directly. We present below the corresponding expression for the case when $n=2$.

Theorem 2: The Pareto boundary for the saturation throughput region of $p$-persistent CSMA when $n=2$ is characterized by the following simple expression:

$$
\sqrt{\frac{T}{\sigma}} \cdot\left(1-S_{1}-S_{2}\right)=2 \sqrt{S_{1} S_{2}} .
$$




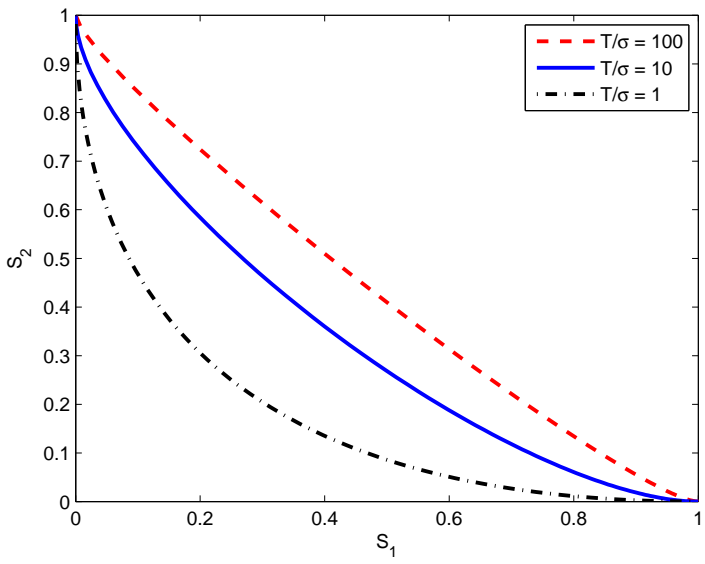

Fig. 1. The boundary of the two-user saturation throughput region for $p$ persistent CSMA

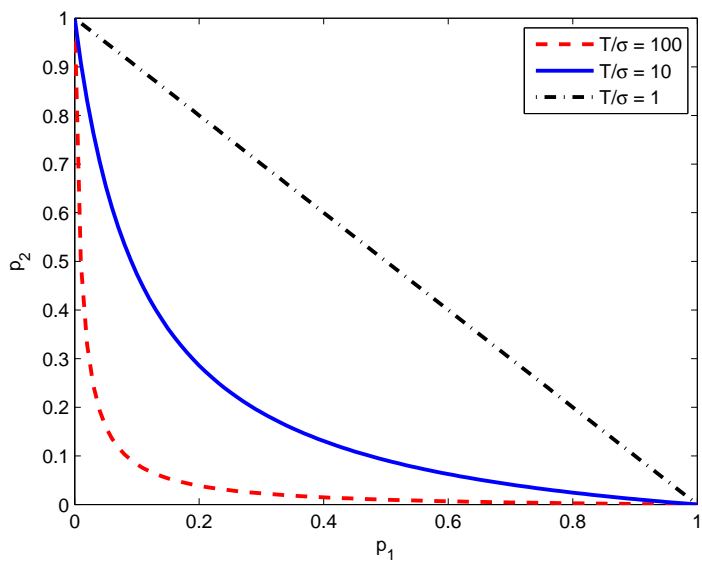

Fig. 2. Access probabilities at the boundary of the two-user saturation throughput region for $p$-persistent CSMA

Note that in the special case of slotted Aloha, when $T=\sigma$, this reduces to the condition $\sqrt{S_{1}}+\sqrt{S_{2}}=1$, as indicated in [16].

Figure 1 shows the throughput region as $\frac{T}{\sigma}$ is varied, based on Theorem 2. The corresponding access probabilities are plotted in Figure 2. We can see in these figures that when $T=\sigma$, the curves do indeed correspond to the known results for slotted Aloha. Interestingly, as the ratio $\frac{T}{\sigma}$ is increased (i.e. longer packets are transmitted), while the saturation throughput region of $p$-persistent CSMA expands and gets closer to the ideal linear region, the access probabilities move farther and farther away from a linear relationship.

\section{REFERENCES}

[1] IEEE $802.11 \quad$ standards. $\quad$ Available from http://standards.ieee.org/getieee802/802.11.html.

[2] IEEE $802.15 .4 \quad$ standards. $\quad$ Available from http://standards.ieee.org/about/get/802/802.15.html.

[3] L. Kleinrock and F. Tobagi, "Packet Switching in Radio Channels: Part I - Carrier Sense Multiple-Access Modes and Their Throughput-Delay Characteristics", IEEE Transactions on Communications, vol. 23, no. 12, pp. 1400-1416, December, 1975.

[4] R. G. Gallager, "A perspective on multiaccess channels", IEEE Transactions on Information Theory, vol. 31, pp. 124-142, 1985.
[5] G. Bianchi, "Performance analysis of the IEEE 802.11 distributed coordination function," IEEE Journal on Selected Areas in Communications, vol. 18, no. 3, pp. 535-547, March, 2000.

[6] F. Calì, M. Conti and E. Gregori, "Dynamic Tuning of the IEEE 802.11 Protocol to Achieve a Theoretical Throughput Limit", IEEE/ACM Transactions on Networking (TON), vol. 8, no. 6, pp. 785-799, December, 2000.

[7] L. Bononi, M. Conti and L. Donatiello, "A Distributed Mechanism for Power Saving in IEEE 802.11 Wireless LANs", ACM/Kluwer Mobile Networks and Applications Journal, vol. 6, no. 3, pp. 211-222, 2001.

[8] V. M. Vishnevsky and A. I. Lyakhov, "IEEE 802.11 Wireless LAN: Saturation Throughput Analysis with Seizing Effect Consideration", Journal of Cluster Computing, vol. 5, no. 2, 2002.

[9] M. Miskowicz, M. Sapor, M. Zych and W. Latawiec, "Performance analysis of predictive p-persistent CSMA protocol for control networks", the 4th IEEE International Workshop on Factory Communication Systems, August, 2002.

[10] R. Bruno, M. Conti, and E. Gregori, "Optimization of efficiency and energy consumption in p-persistent CSMA-based wireless LANs," IEEE Transactions on Mobile Computing, vol. 1, no. 1, pp. 10-31, January/March, 2002.

[11] I. Ramachandran and S. Roy, "Analysis of throughput and energy efficiency of p-persistent CSMA with imperfect carrier sensing", IEEE Global Telecommunications Conference (GLOBECOM '05), December, 2005.

[12] I. Menache and N. Shimkin, "Reservation-based distributed medium access in wireless collision channels", the 3rd International Conference on Performance Evaluation Methodologies and Tools, October, 2008.

[13] H. Inaltekin and S. B. Wicker, "The analysis of Nash equilibria of the one-shot random-access game for wireless networks and the behavior of selfish nodes," IEEE/ACM Transactions on Networking, vol. 16, no. 5, pp. 1094-1107, Oct. 2008.

[14] F. Hsu and H. Su, "Analysis of a CSMA-Based Wireless Network with Heterogeneous Nodes: Feasible Throughput Region and Power Consumption", Arxiv preprint, http://arxiv.org/abs/1003.5771.

[15] N. Abramson, "The ALOHA System - Another Alternative for Computer Communications". Proceedings of 1970 Fall Joint Computer Conference, November, 1970.

[16] J. Massey and P. Mathys, "The collision channel without feedback", IEEE Transactions on Infomation Theory, vol. 31, no. 2, pp. 192-204, March, 1985.

[17] Sheldon M. Ross, "Introduction to Probability Models", 10th Edition, Academic Press, United States of America, 2009. 